\title{
MMME and DME: Two New Eigenvalue-Based Detectors for Spectrum Sensing in Cognitive Radio
}

\author{
Andreas Bollig and Rudolf Mathar \\ Institute for Theoretical Information Technology \\ RWTH Aachen University \\ D-52074 Aachen, Germany \\ Email: \{bollig, mathar\}@ti.rwth-aachen.de
}

\begin{abstract}
Cognitive radio and dynamic spectrum access (DSA) promise to ease the scarcity of radio spectrum, which is growing more acute as the demand for wireless connectivity increases. One of the key ingredients of a reliable and efficient DSA system is spectrum sensing, i.e., the act of checking a spectral resource's occupancy state before opportunistically accessing it. To this end, the present work proposes two new eigenvalue-based detectors, the Maximum-Minus-Minimum-Eigenvalue (MMME) detector and the Difference-of-Means-of-Eigenvalues (DME) detector, both of which exploit the properties of the eigenvalues of the covariance matrix of a received signal, which is contaminated with i.i.d. noise. We explain the intuition behind the new detectors, investigate the choice of the DME detector's parameter and assess their performance in comparison to other covariancebased detectors.

Index Terms-Spectrum Sensing, Eigenvalues, Covariance Matrix, Cognitive Radio
\end{abstract}

\section{INTRODUCTION}

In the recent years, radio spectrum has become a tremendously scarce resource. This is due to a dramatically increased demand for wireless services on the one side and the legacy of spectrum licensing policies from the last decades on the other side. The growing spectral scarcity stands in stark contrast to a continued underutilization of large parts of the licensed spectrum. Many licensees only make use of their share of the spectrum at certain geographic regions or certain points in time, thus creating so called spectrum holes.

In order to make better use of the resources given, opportunistic spectrum access has been proposed as part of the cognitive radio vision [1]. The idea is to let unlicensed transceivers (secondary users) access spectral bands while their licensees (primary users) don't occupy them. To facilitate safe spectrum reuse such that the unlicensed transmission doesn't cause interference in the primary system, the secondary system has to monitor the spectrum for primary user activity reliably and free the spectral resources as soon as the licensee tries to access them. Determining the occupancy status of a spectral band is known as spectrum sensing.

The spectrum sensing problem can be cast as a hypothesis test as follows. Consider a secondary system receiver sampling

This work was partly supported by the Deutsche Forschungsgemeinschaft (DFG) project CoSeCo (grant MA 1184/18-1) and by the UMIC research cluster of the RWTH Aachen University. some frequency band, resulting in the baseband signal $x(t)$. To make a statement about the band's occupancy, it has to be decided which of the following two hypotheses is true.

$$
\begin{array}{llc}
\mathcal{H}_{0}: x(n) & = & \eta(n), \\
\mathcal{H}_{1}: x(n) & = & s(n)+\eta(n),
\end{array}
$$

where $\eta(n)$ denotes receiver noise and $s(n)$ stands for a primary user signal after propagation effects.

To this end, a number of solutions have been proposed in the literature [2]-[4]. Based on the amount of information about the primary user signal incorporated, these solutions can be classified into the following three categories: energy detection, feature detection and matched filter detection. While energy detectors don't make use of any prior knowledge in regard to the primary user's (PU) signal, matched filter detectors need to know the exact waveform of at least part of the signal emitted by the PU, typically a pilot or preamble. Feature detectors are an in-between, as they only make assumptions about structural or statistical properties of the signal.

One class of feature detectors are eigenvalue-based detectors, several of which have been proposed in the literature, see e.g. [5]-[9]. These algorithms exploit the properties of the eigenvalues of a signal's covariance matrix in order to detect primary user activity.

The contribution of this work lies in proposing two new eigenvalue-based detection algorithms, the Maximum-MinusMinimum-Eigenvalue (MMME) detector and the Differenceof-Means-of-Eigenvalues (DME) detector, giving an intuition of their respective inner workings as well as discussing the choice of the second detector's parameter. Subsequently, we analyze their performance in comparison to other known eigenvalue-based detectors via numerical simulation.

The remainder of this work is structured as follows. In section II the system model is layed out. Following that, section III presents some covariance-based detectors, which will be considered in the performance comparison. Section IV contains the proposition of the new detectors and explains the respective intuitions behind them. The numerical evaluation and its results are described in section $\mathrm{V}$, while section VI concludes the work and presents some future research directions. 


\section{System MOdeL}

The system model used in this work is a close adaptation of the model introduced in [6], which is beneficial regarding comparability of the results. For completeness, we shortly introduce it in the following.

Consider a system of $P$ primary users, each transmitting a signal $s_{j}(t)$, with $j \in\{1, \ldots, P\}$. The secondary system receives a noise-contaminated superposition of these primary user signals after the effects of path loss, multipath fading and time dispersion. The noise mixed into the received signal is assumed to be zero-mean, i.i.d. and Gaussian with variance $\sigma^{2}$. The secondary system oversamples the symbols of the PU signal by the factor $M$, i.e., either there is one cognitive radio (CR) possessing $M$ receive antennas or there are $M$ collaborating CRs.

The samples acquired in the discrete time-domain during one symbol duration are given by

$$
\mathbf{x}(n)=\left[x_{1}(n), x_{2}(n), \ldots, x_{M}(n)\right]^{\mathrm{T}}
$$

for the $n$th symbol.

We define the channel between the $j$ th PU and the respective receive antennas of the $\mathrm{CR}$ at the time of symbol $n$ as

$$
\mathbf{h}_{j}(n)=\left[h_{1 j}(n), h_{2 j}(n), \ldots, h_{M j}(n)\right]^{\mathrm{T}},
$$

while the additive noise corrupting the reception of symbol $n$ is denoted by

$$
\boldsymbol{\eta}(n)=\left[\eta_{1}(n), \eta_{2}(n), \ldots, \eta_{M}(n)\right]^{\mathrm{T}} .
$$

We consider sets of $L$ consecutive outputs, which we express as

$$
\begin{aligned}
\hat{\mathbf{x}}(n)= & {\left[\mathbf{x}^{\mathrm{T}}(n), \mathbf{x}^{\mathrm{T}}(n-1), \ldots, \mathbf{x}^{\mathrm{T}}(n-L+1)\right]^{\mathrm{T}}, } \\
\hat{\boldsymbol{\eta}}(n)= & {\left[\boldsymbol{\eta}^{\mathrm{T}}(n), \boldsymbol{\eta}^{\mathrm{T}}(n-1), \ldots, \boldsymbol{\eta}^{\mathrm{T}}(n-L+1)\right]^{\mathrm{T}} \text { and } } \\
\hat{\mathbf{s}}(n)= & {\left[s_{1}(n), s_{1}(n-1), \ldots, s_{1}\left(n-N_{c}-L+1\right), \ldots,\right.} \\
& \left.s_{P}(n), s_{P}(n-1), \ldots, s_{P}\left(n-N_{c}-L+1\right)\right]^{\mathrm{T}},
\end{aligned}
$$

where the $n$th symbol of the $j$ th PU is denoted by $s_{j}(n)$ and $N_{c}$ stands for the order of the channel, i.e. the length of the channel impulse response. $L$ is a so-called smoothing factor.

Given the above definitions, we can write

$$
\hat{\mathbf{x}}(n)=\mathbf{H} \hat{\mathbf{s}}(n)+\hat{\boldsymbol{\eta}}(n),
$$

with $\mathbf{H}=\left[\mathbf{H}_{1}, \mathbf{H}_{2}, \ldots, \mathbf{H}_{P}\right]$, where

$$
\mathbf{H}_{j}=\left[\begin{array}{cccccc}
\mathbf{h}_{j}(0) & \ldots & \ldots & \mathbf{h}_{j}\left(N_{c}\right) & & 0 \\
& \ddots & & & \ddots & \\
0 & & \mathbf{h}_{j}(0) & \ldots & \ldots & \mathbf{h}_{j}\left(N_{c}\right)
\end{array}\right] .
$$

The statistical covariance matrix of the received signal can now be written as

$$
\begin{aligned}
\mathbf{R}_{x} & =\mathrm{E}\left(\hat{\mathbf{x}}(n) \hat{\mathbf{x}}^{\mathrm{H}}(n)\right) \\
& =\mathbf{H R}_{s} \mathbf{H}^{\mathrm{H}}+\mathbf{R}_{\eta} \\
& =\mathbf{H R}_{s} \mathbf{H}^{\mathrm{H}}+\sigma^{2} \mathbf{I},
\end{aligned}
$$

with $\mathbf{R}_{s}=\mathrm{E}\left(\hat{\mathbf{s}}(n) \hat{\mathbf{s}}^{\mathrm{H}}(n)\right)$ and $\mathbf{R}_{\eta}=\mathrm{E}\left(\hat{\boldsymbol{\eta}}(n) \hat{\boldsymbol{\eta}}^{\mathrm{H}}(n)\right)$ being the statistical covariance matrices of the PU signals and the additive noise respectively and $\mathbf{I}$ denoting the identity matrix. Note that the last step in (8) is due to the noise being i.i.d..

Since we are not able to acquire an infinite amount of samples, we cannot come by the statistical covariance matrix and have to settle for its estimation, the $M L \times M L$ sample covariance matrix, which for our signal model is obtained as

$$
\hat{\mathbf{R}}_{x}\left(N_{s}\right)=\frac{1}{N_{S}} \sum_{n=L-1}^{L-2+N} \hat{\mathbf{x}}(n) \hat{\mathbf{x}}^{\mathrm{H}}(n),
$$

with $N_{s}$ being the number of collected symbols.

\section{PRIOR WORK}

Several spectrum sensing algorithms exploiting the properties of the covariance matrix can be found in the literature. Some of these will be employed in a performance evaluation of the two new eigenvalue-based detectors in section V. They will shortly be covered in the following. For each of the detectors, a test-statistic $T$ will be given such that for some threshold $\gamma$, the detector decides on the occupancy status of the spectral resource according to the following test:

$$
T \underset{\mathcal{H}_{0}}{\stackrel{\mathcal{H}_{1}}{\gtrless}} \gamma,
$$

i.e., if the test statistic is higher than the threshold $\gamma$, the detector decides that a PU signal is present. If it is lower, the detector decides that there is no PU signal present.

\section{A. Maximum-Minimum-Eigenvalue (MME) Detector}

We denote the maximum - and minimum eigenvalue of $\hat{\mathbf{R}}_{x}\left(N_{s}\right)$ as $\hat{\lambda}_{\max }$ and $\hat{\lambda}_{\min }$ respectively. The MME detector as introduced in [5] is given by

$$
T_{\text {MME }}=\frac{\hat{\lambda}_{\max }}{\hat{\lambda}_{\min }} \underset{\mathcal{H}_{0}}{\stackrel{\mathcal{H}_{1}}{\gtrless}} \gamma_{\mathrm{MME}} .
$$

\section{B. Maximum-Eigenvalue-Trace (MET) Detector}

The MET detector has initially been introduced in [7]. It can be written as

$$
T_{\text {MET }}=\frac{\hat{\lambda}_{\text {max }}}{\operatorname{Tr}\left(\hat{\mathbf{R}}_{x}\left(N_{s}\right)\right)} \underset{\mathcal{H}_{0}}{\stackrel{\mathcal{H}_{1}}{\gtrless}} \gamma_{\text {MET }},
$$

where $\operatorname{Tr}(\cdot)$ stands for the trace operation.

\section{Cholesky Factorization Squares (CFS) detector}

Considering the Cholesky factorization $\hat{\mathbf{R}}_{x}\left(N_{s}\right)=\hat{\mathbf{Q}}^{\mathrm{T}} \hat{\mathbf{Q}}$, the CFS detector introduced in [8] is defined as

$$
T_{\mathrm{CFS}}=\frac{\sum_{1 \leq i \leq j \leq M L} \hat{q}_{i j}^{2}}{\sum_{1 \leq i \leq M L} \hat{q}_{i i}^{2}} \underset{\mathcal{H}_{0}}{\stackrel{\mathcal{H}_{1}}{\gtrless}} \gamma_{\mathrm{CFS}},
$$

where $\hat{q}_{i j}$ is the $(i, j)$ th element of $\hat{\mathbf{Q}}$. 


\section{Quadratic Sphericity Test (QST)}

Given the ordered eigenvalues of $\hat{\mathbf{R}}_{x}\left(N_{s}\right)$ as

$$
\hat{\lambda}_{\max }=\hat{\lambda}_{1} \geq \hat{\lambda}_{2} \geq \cdots \geq \hat{\lambda}_{M L}=\hat{\lambda}_{\text {min }},
$$

the QST as described in [9] is given as

$$
T_{\mathrm{QST}}=\frac{\sqrt{\sum_{1 \leq i \leq M L} \hat{\lambda}_{i}^{2}}}{\sum_{1 \leq i \leq M L} \hat{\lambda}_{i}} \underset{\mathcal{H}_{0}}{\gtrless} \gamma_{\mathrm{QST}} .
$$

\section{TWO NEW EIGENVALUE-BASED DETECTORS}

\section{A. Maximum-Minus-Minimum-Eigenvalue (MMME) Detector}

Consider the ordered eigenvalues $\lambda_{1}, \ldots, \lambda_{M L}$ of the statistical covariance matrix $\mathbf{R}_{x}$ as well as the ordered eigenvalues $\rho_{1}, \ldots, \rho_{M L}$ of $\mathbf{H R}_{s} \mathbf{H}^{\mathrm{H}}$ analogous to (14). The MME detector (11) is based on the idea that since $\lambda_{n}=\rho_{n}+\sigma^{2}$, it holds that $\frac{\lambda_{1}}{\lambda_{M L}}=1$ for $\mathcal{H}_{0}$ (if no PU signal is present, $\rho_{i}=\left.0\right|_{i=1} ^{M L}$ ) and $\frac{\lambda_{1}}{\lambda_{M L}}>1$ for $\mathcal{H}_{1}$.

As the authors of [6] find, choosing $L$ large enough leads to $\rho_{M L}=0$ even if a PU signal is present. Taking this into account and including the estimation noise caused by the finite amount of samples gathered, we can express the detector's test statistic as

$$
\begin{aligned}
& T_{\mathrm{MME}, \mathcal{H}_{1}}=\frac{\hat{\lambda}_{1}}{\hat{\lambda}_{M L}}=\frac{\rho_{1}+\sigma^{2}+\epsilon_{1}}{\sigma^{2}+\epsilon_{2}}=\frac{\sigma^{2}+\epsilon_{1}}{\sigma^{2}+\epsilon_{2}}+\frac{\rho_{1}}{\sigma^{2}+\epsilon_{2}}, \\
& T_{\mathrm{MME}, \mathcal{H}_{0}}=\frac{\hat{\lambda}_{1}}{\hat{\lambda}_{M L}}=\frac{\sigma^{2}+\epsilon_{3}}{\sigma^{2}+\epsilon_{4}},
\end{aligned}
$$

where $-\epsilon \leq \epsilon_{i} \leq\left.\epsilon\right|_{i \in\{1,2,3,4\}}$ models the perturbation of the eigenvalues. The estimation noise vanishes for high $N_{s}$, i.e., $\lim _{N_{s} \rightarrow \infty} \epsilon=0$. Thus, we can see that on top of the estimation noise, the MME detector is affected by the SNR. The lower the SNR, the lower $\rho_{1} /\left(\sigma^{2}+\epsilon_{2}\right)$.

To tackle this disadvantage, we propose the MMME detector as a modification of the MME detector. Its test statistic is given as

$$
T_{\mathrm{MMME}}=\ln \left(\frac{e^{\hat{\lambda}_{\text {max }}}}{e^{\hat{\lambda}_{\text {min }}}}\right)=\hat{\lambda}_{\text {max }}-\hat{\lambda}_{\text {min }} \underset{\mathcal{H}_{0}}{\stackrel{\mathcal{H}_{1}}{\gtrless}} \gamma_{\text {MMME }}
$$

Note that the $\ln (\cdot)$ has no influence on the detector's performance. Investigating this detector, we find that

$$
\begin{aligned}
& T_{\mathrm{MMME}, \mathcal{H}_{1}}=\ln \left(\frac{e^{\hat{\lambda}_{1}}}{e^{\hat{\lambda}_{M L}}}\right)=\ln \left(\frac{e^{\rho_{1}+\sigma^{2}+\epsilon_{1}}}{e^{\sigma^{2}+\epsilon_{2}}}\right)=\rho_{1}+\epsilon_{1}-\epsilon_{2}, \\
& T_{\mathrm{MMME}, \mathcal{H}_{0}}=\ln \left(\frac{e^{\hat{\lambda}_{1}}}{e^{\hat{\lambda}_{M L}}}\right)=\ln \left(\frac{e^{\sigma^{2}+\epsilon_{3}}}{e^{\sigma^{2}+\epsilon_{4}}}\right)=\epsilon_{3}-\epsilon_{4},
\end{aligned}
$$

which shows that asymptotically, the detector is independent of the SNR.

\section{B. Difference-of-Means-of-Eigenvalues (DME) detector}

A typical strategy to diminish noise is to average over multiple values. Neither the MME -, nor the MMME detector exploits this. Both only make use of two of the $M L$ available eigenvalues. Building on the idea of the MMME detector, we implement this concept by proposing the DME detector as

$$
T_{\mathrm{DME}}\left(N_{1}\right)=\frac{1}{N_{1}} \sum_{i=1}^{N_{1}} \hat{\lambda}_{i}-\frac{1}{M L-N_{1}} \sum_{i=N_{1}+1}^{M L} \hat{\lambda}_{i} \underset{\mathcal{H}_{0}}{\stackrel{\mathcal{H}_{1}}{\gtrless}} \gamma_{\mathrm{DME}} .
$$

The choice of $N_{1}$ comes down to a tradeoff between multiple objectives. In order to achieve a good detection performance, it would generally be favorable to minimize the overlap between the PDF of $T_{\mathrm{DME}, \mathcal{H}_{0}}$ and the PDF of $T_{\mathrm{DME}, \mathcal{H}_{1}}$. In fact, if the overlap of the two PDFs was zero and a way of setting the correct decision threshold was known, perfect detection could be achieved. When setting $N_{1}$, we are thus interested in minimizing the mean of $T_{\mathrm{DME}, \mathcal{H}_{0}}$ as well as maximizing the mean of $T_{\mathrm{DME}, \mathcal{H}_{1}}$. We should also try to minimize the respective variances of $T_{\mathrm{DME}, \mathcal{H}_{0}}$ and $T_{\mathrm{DME}, \mathcal{H}_{1}}$. An empirical evaluation of these objectives is given in section $\mathrm{V}$.

\section{Numerical Evaluation}

For the simulation, we consider a scenario exhibiting the parameters given in table I. For each of the Monte Carlo realizations, a random BPSK signal for each PU, a random Gaussian distributed channel and random noise is generated.

TABLE I

SCENARIO PARAMETERS

\begin{tabular}{lcc}
\hline Parameter & Symbol & Value(s) \\
\hline Number of PUs & $P$ & 2 \\
Number of receive antennas / collab. CRs & $M$ & 4 \\
Channel order & $N_{c}$ & 9 \\
Smoothing factor & $L$ & 8 \\
Number of symbols & $N_{s}$ & $10^{5}$ \\
Number of Monte Carlo realizations & & $10^{4}$ \\
\hline
\end{tabular}

In figure 1 , the mean of $T_{\mathrm{DME}}$ over the Monte Carlo realizations is plotted for different SNRs in the case where a PU signal is present $\left(\mathcal{H}_{1}\right)$ as well as in the case where no PU signal is present $\left(\mathcal{H}_{0}\right)$. Recall that while making the choice of $N_{1}$ we try to minimize $T_{\mathrm{DME}}$ in the case of $\mathcal{H}_{0}$, but try to maximize it in the case of $\mathcal{H}_{1}$.

What we can see in figure 1 is that the lower the SNR, the closer the $\mathcal{H}_{1}$-curve comes to the $\mathcal{H}_{0}$-curve. We also see, that the lower the SNR, the more closely the $\mathcal{H}_{1}$-curve resembles the $\mathcal{H}_{0}$-curve in its shape. These effects are brought along by the decreasing influence of the signal eigenvalues in the test statistic caused by a decreasing SNR. The figure gives us a clear picture of the forces, which have to be traded off against each other. In order to minimize $T_{\mathrm{DME}, \mathcal{H}_{0}}, N_{1}$ would have to be chosen to be roughly $\frac{M L}{2}$. However, even for low SNRs $N_{1}$ has to be set to 1 to maximize $T_{\mathrm{DME}, \mathcal{H}_{1}}$.

As we can see in figure 2 , minimizing the variance of $T_{\mathrm{DME}, \mathcal{H}_{0}}$ can again be achieved by choosing $N_{1} \approx \frac{M L}{2}$. Regarding the minimization of the variance of $T_{\mathrm{DME}, \mathcal{H}_{1}}$, the 


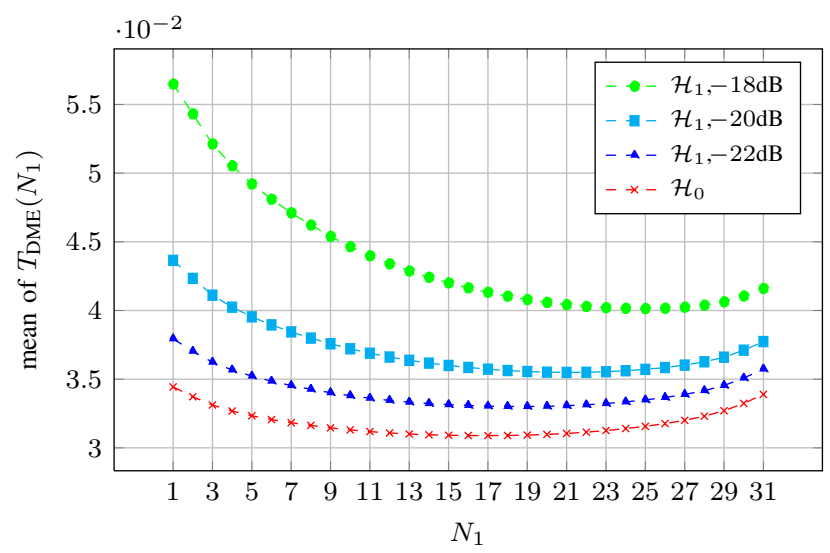

Fig. 1. Mean of $T_{\mathrm{DME}}\left(N_{1}\right)$ over $10^{4}$ Monte Carlo realizations for different SNRs in the case of a present PU signal $\left(\mathcal{H}_{1}\right)$ as well as in the case of noise only $\left(\mathcal{H}_{0}\right)$.

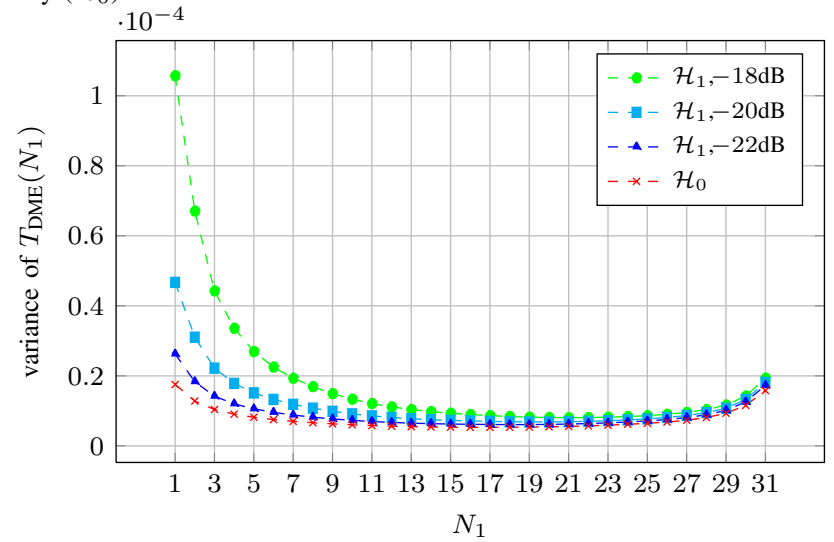

Fig. 2. Variance of $T_{\mathrm{DME}}\left(N_{1}\right)$ over $10^{4}$ Monte Carlo realizations for different SNRs in the case of a present PU signal $\left(\mathcal{H}_{1}\right)$ as well as in the case of noise only $\left(\mathcal{H}_{0}\right)$.

higher the SNR, the higher $N_{1}$ has to be chosen. For very low SNRs it is approximately the same as for $T_{\mathrm{DME}, \mathcal{H}_{0}}$.

The right choice of $N_{1}$ has a large effect on the performance of the DME detector. Thus, it would be advantageous to have an analytic way of setting it optimally.

In figure 3, the performance of the two new detectors is compared against other detectors from the literature. For the scenario used in the simulation, the optimal choice of $N_{1}$ empirically turned out to be 8 . The figure shows the receiver operating characteristic (ROC) of the detectors at an SNR of $-20 \mathrm{~dB}$. We observe, that the DME detector shows the highest probability of detection $\left(P_{\mathrm{d}}\right)$ for all probabilities of false alarm $\left(P_{\mathrm{fa}}\right)$. What can also be seen, is that although the MMME detector is weaker than the top contenders, it clearly outperforms the MME detector.

\section{CONCLUSION}

In this work, we have introduced two new eigenvaluebased detectors for spectrum sensing, the Maximum-MinusMinimum-Eigenvalue (MMME) detector and the Differenceof-Means-of-Eigenvalues (DME) detector. We have discussed the respective ideas behind them and have investigated the tradeoff leading to an optimal parameter choice for the DME test statistic. Finally, we have numerically evaluated

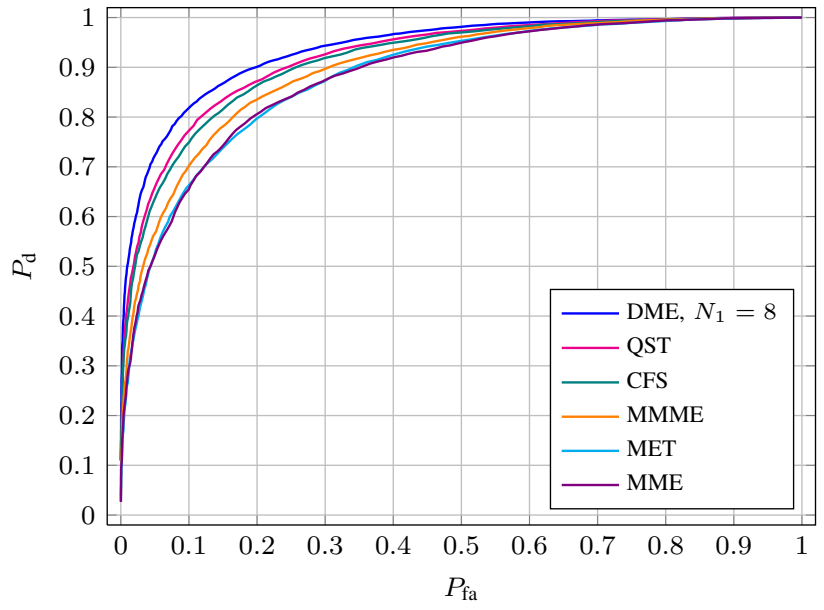

Fig. 3. Receiver operating characteristics of DME (19) with $N_{1}=8$, QST (15), CFS (13), MMME (17), MET (12) and MME (11) at an SNR of -20dB.

the performance of the two new detectors in comparison to other detectors. Based on the MME detector, the MMME detector improves upon the performance of its origin, while the DME detector shows the best performance of all considered detectors.

Since the choice of the DME detector's parameter heavily influences its performance, an interesting research direction would be to find an analytic way of obtaining the optimal parameter. It would also be interesting to find an analytic expression for the new detectors' thresholds based on a desired false alarm rate.

\section{REFERENCES}

[1] S. Haykin, "Cognitive radio: Brain-empowered wireless communications," IEEE Journal on Selected Areas in Communications, vol. 23, no. 2, pp. 201-220, Feb. 2005.

[2] T. Yücek and H. Arslan, "A survey of spectrum sensing algorithms for cognitive radio applications," IEEE Communications Surveys Tutorials, vol. 11, no. 1, pp. $116-130$, Mar. 2009.

[3] Y. Zeng, Y.-C. Liang, A. T. Hoang, and R. Zhang, "A review on spectrum sensing for cognitive radio: challenges and solutions," EURASIP Journal on Advances in Signal Processing, vol. 2010, Jan. 2010.

[4] E. Axell, G. Leus, E. Larsson, and H. Poor, "Spectrum sensing for cognitive radio : State-of-the-art and recent advances," IEEE Signal Processing Magazine, vol. 29, no. 3, pp. 101-116, May 2012.

[5] Y. Zeng and Y.-C. Liang, "Maximum-minimum eigenvalue detection for cognitive radio," in IEEE 18th International Symposium on Personal, Indoor and Mobile Radio Communications (PIMRC), 2007., Sep. 2007, pp. $1-5$.

[6] — , "Eigenvalue-based spectrum sensing algorithms for cognitive radio," IEEE Transactions on Communications, vol. 57, no. 6, p. 1784-1793, Jun. 2009.

[7] Y. Zeng, Y.-C. Liang, and R. Zhang, "Blindly combined energy detection for spectrum sensing in cognitive radio," IEEE Signal Processing Letters, vol. 15, pp. 649-652, Oct. 2008.

[8] X. Yang, K. Lei, S. Peng, and X. Cao, "Blind detection for primary user based on the sample covariance matrix in cognitive radio," IEEE Communications Letters, vol. 15, no. 1, pp. 40-42, Jan. 2011.

[9] J. Font-Segura, J. Riba, J. Villares, and G. Vazquez, "Quadratic sphericity test for blind detection over time-varying frequency-selective fading channels," in Proceedings of the 38th International Conference on Acoustics, Speech, and Signal Processing (ICASSP 2013), May 2013, pp. 47084712 . 